\title{
Changes of Japanese Phonetics
}

\author{
He Wang \\ School of Foreign Studies,Xi'an University,Xi'an,710065,China
}

\begin{abstract}
Keywords: Changes, Japanese Phonetics, Basic Knowledge
Abstract. In this era of globalization, only learn our own language is not good enough to adapt it. Although the relationship between Japan and China is tense, Japanese and Chinese are inextricably linked to each other. Japanese as part of the language is also a kind of culture, so that we should get to know it. This paper talks about the changes and basic knowledge of Japanese phonetics and how to learn Japanese well.
\end{abstract}

\section{Development of Japanese in China}

Relationship between Japanese and Chinese. The appearance of language plays a very important role in the development of human civilization and social economy. It is an expression method for human beings to communicate ideas. In human society, languages affect each other and no single language can exist alone. When affected by other languages, only by continuous innovation can it survive longer .

Japan has a complex relationship of "similar culture and similar race" with China for a long time. Regardless of racial issues, they have original relations between language and writing. The Chinese QianZiWen was spread to Japan by Dr. Wang Ren and it has a history of one thousand years. During the period of Chinese opening to the outside world, many cultures of Japan entered the life of Chinese. The language also influenced China and made Chinese language more diversified and modernized.

Development of Japanese in China. According to the records, Japan only have language but no character before Chinese characters was spread into. At the beginning of seventh century, Japan borrowed the particularity of Chinese characters and created a mark Japanese pronunciation of "manyokana", that is to say, the transformation of Chinese characters. Before nineteenth century, Chinese affects Japanese. Until 1868 the shogunate stepped down and the MingZhi government began to open its door to the outside world, which make Japan a huge leap forward. At that time, China began to learn from Japan, translating Japanese books and bringing in knowledge of all fields.

In recent years, a trend of learning Japanese was set off in China. Japanese in China is known as "rare foreign language". Since December of 1999, only 150 of all Chinese colleges and universities set Japanese major. While the number was 256 in 2005 and the number is 660 in 2012. Japanese is growing rapidly in China. Although the relationship between Japan and China is tense, more and more people are learning Japanese and even studying overseas in Japan.

\section{Introduction of Japanese Phonetics}

Characteristics of Japanese Pronunciation. To learn Japanese well, we must first understand the characteristics of Japanese pronunciation. Japanese pronunciation is not difficult, because it has simple syllables. A natural unit of speech, also called a beat, is a phonetic unit consisting of one or several phonemes. There are a few differences between Japanese and Chinese. In Japanese, a kana is a syllable and a syllable can be written as a kana (except fist name ). In addition, the vowel compositing the Japanese phoneme is simple and easy to pronounce because there are only five vowels. Japanese pronunciation is simple, so that many people say that the Japanese can speak not mouth. Aspirated and unaspirated are the two kanas in Japanese. The aspirated occurs with the throat relaxed, but there will be a strong flow of air out of mouth. While read unaspirated, throat tension strongly, but air emitted from the mouth is relatively weak. When Chinese learn Japanese, they are easy to confuse the unvoiced and voiced sound. When read promote tone, pronunciation time is often not long enough. 
Tone and Intonation of Japanese. If want to speak fluent Japanese, you should not only understand the characteristics of its pronunciation, but also be familiar with the characteristics of Japanese tone and intonation. "Tone" refers to the level of sound in each word or phrase has a certain degree of distribution. The common tone in Japanese is called "high-low tone". The tone of Japanese can be divided into two types. One is that the first beat is low then the second began to rise but not down. The other is the tone will fall down after second beat, or the first beat rise and then fall down from the second. Both Japanese and other languages have their own "tense". The so-called "tense" is a grammatical category that shows the relationship between the action of predicate verb and the person or thing in the supplement. In Japanese, tense showed by auxiliary verb followed behind the predicate verb. It is mainly divided into: passive voice, possibility modal, spontaneous tense and causative tense, etc.

\section{Pronunciation Position and Method of Japanese Phoneme}

Learning Method of Japanese Phoneme. The most common method of learning Japanese phoneme is to learn fifty-tone figure. Actually, the number of kana in fifty-tone figure is 47 . The pronunciation of vowel and consonant is similar to the international phonetic alphabet. Only few need to pay special attention to.

Learning Japanese phoneme pronunciation, you can take the two commonly used methods. Beginners will use representation to help memorize pronunciation. In the absence of audible voice, the real situation only can be speculated through this method. There is another method commonly used in international phonetic alphabet, but this method is too professional and needs a deep understanding of it and it is not the best method to learn Japanese. The widely used method is roman alphabet notation in the study of Japanese pronunciation. That is to say, use the 26 letters in English to represent Japanese vowels and consonants.

Pronunciation Organs of Japanese Phonetics. Accurate pronunciation needs the help of all parts of the body. The human body does not have a professional organ for sound. The human's pronunciation is achieved through a part of the intentional organ and the digesting system. The part above the throat is called vocal tract and the interface of laryngeal and tracheal called glottis. In the practice of the vowel, the first step is to fall diaphragm, expand the volume of the chest and make lungs to absorb enough air. And then rise diaphragm, so that the lungs exhaled air. When glottis closes, it repeating the action under the impact of the air to make air shock, and then appears original voice. The voice created by vocal cords is weak and monotonous, which need to be amplified and adjusted to become the actual use of voice.

Pronunciation Method of Japanese Vowels. In Japanese pronunciation, it is very important to learn the pronunciation of vowels. If a vowel is not pronounced accurately, the syllable associated with the vowel will not be accurate too. It requires relevant organs (including mouth and tongue) stable when read vowels, and the shape of vocal tract can' $t$ be changed half-way. The stability of Japanese vowels also showed as below: No matter how the voice environment changes, timbre of vowels has not changes significantly.

In Japanese, lips and tongue muscles should relax when read "a", and do not open your mouth too much. In the language flow, try to maintain the relative stability of sound. "i" need vocal organs maintain the natural state of relaxation. "u" also need vocal organs maintain the natural state of relaxation, and lips and tongue muscles should not be too hard. Forms of natural lip. "e" need lips natural expansion. Meanwhile, mouth should not open to both sides and not deliberately folded inward. And "o" requires mouth inward contracting into a flat oval when speak. Having a good command of pronunciation of vowels, the pronunciation of consonants will be much easier.

\section{Japanese Beat}

Origin of Japanese Beat. In the study of Japanese phonetics, syllables and beats are included. They are units of phonematics and phonology respectively. Although there are many similarities in the 
specific performance, they are different concepts in essence. The syllable in Japanese is a unit which is divided according to the theory of phonematics, and it has more theoretical value. However, the beat is more practical than syllable seen from the application point of view.

In order to avoid confusion with the syllables, Kindaichi Haruhiko pointed out that beat belongs to the phonology unit and should be distinguished from the syllables. In the history of Japan, Mr Kamei filial piety is the first person to use beat and take the beat as an important concept in the study of phonology. Kindaichi Haruhiko valued and advocated to use beat, and confirmed the important position of beat in Japanese phonetics according to demand of practical life. He summed up that there are five kinds structure of Japanese beat as below: vowel phoneme, semi vowel phoneme + vowel phoneme, consonant phoneme + vowel phoneme, consonant phoneme + semi vowel phoneme + vowel phoneme, and dial tone and promote tone and long sound of special phoneme. That is to say, every kana has its corresponding beat.

Japanese Syllable. A Japanese scholar pointed out that Japanese syllable is corresponding to each kana. Japanese syllables is a complete phonetic unit represented by kana(except fist name). Each or two words in a row is a syllable. In Japanese, difficult tone, dial tone, promote tone and long tone is respectively regarded as a syllable. There are also other scholars defining syllable based on the time, that is to say, phonetic unit shares same time of pronunciation called syllable.

Syllables can be used in any language. As a part of the world language, the existence of syllables in Japanese is inevitable. As the rhythm academic language in the music field, beat can also solve many problems of syllables in the Japanese. The role of the expression in the Japanese determines the necessity of its existence. As a specific expression of Japanese national language awareness, beat is responsible for the important task of distinguishing the meaning of language in Japanese.

Characteristics of Japanese Beat and Syllable. One syllable in phonematics is divided into two beats, and then divide into different linguistic meanings according to the difference of beat. The beat is a special unit used to distinct linguistic meaning that determined by Japanese according to their own national language awareness and the structural characteristics of syllables. It is this special function that determines the convenience and superiority of beat in the practical application.

Beat is isochronous, which is the best means to distinguish syllable and beat into two different concepts. Beat is expressed and established relying on the equal length of time. But the length of time can not be used to judge the syllable because the syllable is differentiated by length of the pronunciation. There is no length of time division because beat is isochronous. The isochronous characteristics and recognition feature of beat determine its special position in Japanese. The structure of beat is much simpler than that of syllable. All beat of Japanese is open syllable.

\section{Rules of Tone in Japanese}

Introduction of Japanese Tone. In a word or phrase, different height and intensity of each beat makes tone. There are two types of tone, high-low and strong-weak.

Although there are also changes in strength of Japanese beat, mainly some beat is high, some beat is low, so that Japanese tone is high-low type.

The tone of Japanese is high-low type, from high to low or from low to high. A kana represents a beat, including voiceless sound, voiced sound, semi voiced sound and promote tone, dial tone and long tone. However it exclude the small "ya", "yu" yo ", that is to say, a fist name is regarded as a beat, such as "kyu" is one beat not two. While "kyu u" and "ku u" and other long tone are two beats. Japanese consider Tokyo tone as the standard pronunciation, and its tone can be divided into the following several types: Type $0,1,2,3,4,5,6$ and 7 etc. High tone means accent, low tone means schwa.

Characteristics of Japanese Tone. Some words have two or more different tone types, which means these words have different pronunciation in different situations. Some words have two or more connected accents (i.e, symbols of tone type) within a word, which indicating that the word has two or more accents. For example, the tone type of "se i ha $\mathrm{n}$ go u (positive and negative)" is (1)-(1)-(1) type, which means that this word has three accents. That is to say, "se", "ha", and "go" are high tone(accent). 
The accent of Japanese can not be placed separately. That is to say, a word (including follow-up auxiliary) only has these tone configurations as showed below: "high-low-low", "low-high-low" and "low-high-high", and absolutely has no "high-low-high", "low-high-low-high", or "high-low-high-low" etc tone configurations. No matter how long the word is, the tone configuration must conform to this rule.

\section{Japanese Intonation}

Each word has its own tone, sentence also has cadence. We call this cadence intonation.

The intonation of phrases and declarative sentences. In Chinese, each word has its own tone, and it is less affected by other factors in the sentence. In Japanese, whether it is a phrase or a declarative sentence, it always start high and then gradually reduced. Let's look at the tone of phrase first.

In the Mandarin, the tone of "Ni" at the beginning of a sentence is not higher than the "Sha "of the sentence end. When read individual words, the tone of words are kept at the same height. However, when forming a phrase, the tone changes as follows: first keep its original tone and then change according to the principle of "begin with high tone and decrease gradually" . If the height of pronunciation is same, it means special emphasis on a certain meaning, such as "isn't your bag, but the book." Conversely, enhance the tone at the place where you want to emphasize. Pay attention to this when practice.

Intonation of End of Interrogative Sentence. Intonation need to rise at the end of interrogative sentence. The intonation of $\lceil$ de su ka $\rfloor$ in $\lceil$ ko le ha ho $\mathrm{n}$ de su ka $\rfloor$ need to rise when show doubt. While the tone of $\lceil$ de su ka $\rfloor$ in $\lceil$ ko le ha ho n de su ka $\rfloor$ need to reduce when show confirm. Normally, people will not say 「de su ka $」$ and only say $\lceil$ ho n?」(Is it a book?). 「ho n.」(Yes, it is.). Seeing from this sentence, you can find the importance of intonation. However, the tone of $\lceil$ ho $\mathrm{n}\rfloor$ should be maintained. In this context, enhance the tone of $\lceil$ ho $\mathrm{n}$ ? 」

Besides, 「na n de su ka.」(What is that?) shows the common doubt. However, 「na n de su ka?」 (What is the hell that?) shows the blame to others.

\section{Summary}

As described above, intonation plays an important role in defining the meaning of sentence and natural pronunciation. Therefore, it is necessary to listen to the conversation in recording repeatedly and do imitate practice. You must pay attention to the changes of intonation of the sentence when practice.

In Chinese, there are many dialects, which are different from Mandarin in vocabulary and pronunciation. The Japanese also have dialects, although they are different from standard Japanese, the difference is much smaller than Chinese. As long as you learn basic tone and intonation well, your Japanese could be understood no matter where the Japanese come from.

\section{References}

[1] Wenzhu Bian, Discuss the Role of Film Used in Japanese Teaching[J], New Course Learning(middle), 25, 2011.

[2] Xin Wang, Comparative Study on Negative Representations between Chinese and Japanese[D], Jilin University, 89, 2011.

[3] Dawei He, Discussion of Limited Relationship between Japanese Predicate and Personal Subject[D], Heilongjiang University, 115, 2011.

[4] Junqin Jia, Cultural Introduction in Japanese Teaching[J], Journal of Lishui University, 75, 2008.

[5] Bo Li, A Contrastive Study of Chinese and Japanese Word Order from the Perspective of Linguistic Typology[D], Shanghai International Studies University, 56, 2011. 
[6] Zheng Kong, A Study of Japanese Training Course in Society, Hunan University, 95, 2013. 